\title{
The Effect of the Tilted Field in the Fractional Quantum Hall Systems: Numerical Studies for the Solid-liquid Transition
}

\author{
Yue $\mathrm{Yu}^{1}$ and Shijie Yang ${ }^{2}$ \\ ${ }^{1}$ Institute of Theoretical Physics, Chinese Academy of Sciences, P.O. Box 2735, Beijing 100080, China \\ 2 Center for Advanced Study, Tsinghua University, Beijing 100084, China
}

(October 30, 2018)

\begin{abstract}
We construct a generalized Laughlin-liquid wave function and a variational electron solid wave function when the magnetic field is tilted. The energy of the liquid state is evaluated by Monte Carlo methods while the energy of the solid state is calculated by the optimization. Comparing these two energies for a given tilted angle $\theta$, it is seen that the critical filling factor $\nu_{c}$ of $\theta$ of the solid-liquid transition increases as the tilted angle. The implication to the experiment is that: i) the insulating phase may harder be melt as $\nu \rightarrow 1 / 5$ such that the width of the valley of the longitudinal resistance may become narrow as the filed is tilted; ii) it is expected that even in the vicinity of $\nu=1 / 3$ for the electron system in the presence of the tilted field, the insulating phase may be observed.
\end{abstract}

PACS numbers: 73.20.Dx,73.40.Kp,71.45.Nt,

After the fractional quantum Hall effects (FQHE) [1] was revealed, the measurement in a tilted field has become an established technique. Mostly, the tiled field is used to explore the spin related effects [2]. It also affected the transport of the bilayer system due to the in-plane field couples to the psuedospin degrees of freedom [3]. The high Landau level stripe phase is strongly influenced by the tilted field $\llbracket$. For the spin polarized system, the tilted field effects are produced by either the finite thickness of the layer [5] or the subband Laudau level coupling [6]. For the later case, only a numerical diagonalization for a small system was carried out. One of focuses in the present work is trying to construct a Laughlin-type many-particle wave function when the magnetic field is tilted.

In the early days that Laughlin's wave function was established, a set of the numerical works to check Laughlin's original wave function was presented [7 9]. Compared to the energy of the electron solid state, which is estimated by a variational method [10, it was found that the critical filling factor $\nu_{c}$ is around $\frac{1}{6.5}$. Considering the Landau level mixing, it was said that the electron solid state may appear in a much larger filling factor [11, 12]. The finite layer thickness can soften the short range behavior of the Coulomb interaction and thus leads to favor the competing electron solid [13,11, 14]. However, at the exact $\nu=1 / m$, the Laughlin-Jastrow correlation for the electron solid may leads to the melt of the electron solid 115. while the energy of the liquid state at an arbitrary filling factor is higher than that from the interpolating between the odd-denominator filling factor [16], which may explain an reentrant insulating phase near $\nu=1 / 5$ for the electron [17] and $\nu=1 / 3$ for the hole [11].

In this work, we plan to study the competition between the solid and liquid states as vary of the tilted field through the subband Landau level coupling. We perform Monte Carlo simulations for a generalized Laughlin state in the presence of the tilted field. As expected, the liquid state energy is lowered as increasing the tilted an- gle. Meanwhile, we use a variational wave function for the solid state in the tilted field and optimize the corresponding energy. The solid state energy is also lowered as increasing the tilted angle, whose magnitude lowered is always larger than that of liquid state energy. We can see that the critical filling factor for the solid-liquid transition may be considerably lifted. In this work, we do not consider the Laughlin-Jastrow correlation to the electron solid yet, which leads to an instability of the electron solid exactly at $\nu=1 / m$ for $m$ an odd integer [15]. Thus, although the tilted field effect may not destroy the liquid state at the exact filling factor $1 / 5$, the insulatorquantum Hall liquid transition may become harder to be melt as $\nu \rightarrow 1 / 5$ such that the width of the valley of the longitudinal resistance may be narrowed as the tilted angle increases. Taking the energy lowered by the Laudau level mixing in the zero tilted field [12] as an estimation, one may even expect that the insulating phase will be observed near $\nu=1 / 3$ for the electron systems in the presence of the tilted field.

To consider the subband effect, we assume a quasi-twodimensional electron gas confined by a harmonic potential with $V(z)=\frac{1}{2} m_{e} \Omega^{2} z^{2}$, where $m_{e}$ is the electron band mass and $\Omega$ is the frequency of the harmonic potential in the perpendicular direction $z$. The applied magnetic field is tilted by an angle $\theta$ to the two-dimensional $x-y$ plane. The single-particle problem has been solved in literature 18,19. To construct a many-particle Laughlin-type state for the convenience of the numerical calculation, we take an alternative way. Working in the symmetric gauge, although the rotational symmetry of two dimensions is broken by the in-plane field, there is still a conserved quantity, $L_{\xi}=b_{\xi}^{\dagger} b_{\xi}$ no matter whether the magnetic field is tilted, where $\xi=x+i y$ and $b_{\xi}^{\dagger}=\frac{1}{\sqrt{2}}\left(-\partial_{\bar{\xi}}+\frac{1}{2} \xi\right)$ is the generator of the magnetic translation. The eigen state of $L_{\xi}$ thus is the same as that in $B_{\|}=0$. The eigen state of the single-particle Hamiltonian for $B_{\|} \neq 0$, then, can be the linear combination of the degenerate states with 
a given eigen value $m_{L}$ of $L_{\xi}$,

$$
\begin{aligned}
& \Phi_{m_{L}}(\vec{r})=\sum_{n_{1}, n_{2}} C_{n_{1} n_{2}}^{\tilde{n}_{1} \tilde{n}_{2}} \Psi_{n_{1} n_{2} m_{L}}(\vec{r}), \\
& \Psi_{n_{1} n_{2} m_{L}}(\vec{r})=f_{n_{1} n_{2} m_{L}}(\vec{r}) e^{-\frac{1}{4}|\xi|^{2}-\frac{1}{2} z^{\prime 2}} \\
& \propto a_{\xi}^{\dagger n_{1}} a_{z}^{\dagger n_{2}} b_{\xi}^{\dagger m_{L}} e^{-\frac{1}{4}|\xi|^{2}-\frac{1}{2} z^{\prime 2}},
\end{aligned}
$$

where the unit of the magnetic length $l_{B}=\sqrt{\frac{c \hbar}{e B_{\perp}}}=1$ has been used and $a_{\xi}^{\dagger}=\frac{1}{\sqrt{2}}\left(-\partial_{\xi}+\frac{1}{2} \bar{\xi}\right)$ and $a_{z}^{\dagger}=$ $\frac{1}{\sqrt{2}}\left(-\partial_{z^{\prime}}+z^{\prime}\right)$ are the harmonic operators with the frequencies $\omega_{c}=\frac{e B_{\perp}}{m_{e} c}$ and $\Omega$, respectively; $z^{\prime}=\hat{\Omega}^{1 / 2} z$ with $\hat{\Omega}=\tilde{\Omega} / \omega_{c}, \tilde{\Omega}^{2}=\Omega^{2}+\omega_{\|}^{2}$ and $\omega_{\|}==\frac{e B_{\|}}{m_{e} c}$. The combination constants $C_{n_{1} n_{2}}^{\tilde{n}_{1} \tilde{n}_{2}}$ can be determined by a standard method to find the common eigen states of two commutated operators. For the ground state, one denotes $C_{n_{1} n_{2}}^{00}=C_{n_{1} n_{2}}$, for example, $C_{10}=C_{01}=0$ and

$$
C_{11}=-\frac{\omega_{c}+\tilde{\Omega}-\omega_{+}-\omega_{-}}{\tilde{\omega}} C_{00},
$$

for $C_{00}$ is determined by the normalized condition. Other $C_{n_{1} n_{2}}$ can be deduced by recursive relations. Here $\omega_{ \pm}^{2}=\frac{1}{2}\left(\tilde{\Omega}^{2}+\omega_{c}^{2}\right) \pm \frac{1}{2} \sqrt{\left(\tilde{\Omega}^{2}-\omega_{c}^{2}\right)^{2}+4 \tilde{\omega}^{2} \tilde{\Omega} \omega_{c}}$ are the corresponding frequencies to the diagonalized harmonic operators.

For the liquid state, following Laughlin's track in writing down his wave function, the required incompressible wave function for the odd denominator filling factor $\nu=1 / m$ has the form

$$
\begin{aligned}
\Psi_{m}\left(\vec{r}_{1}, \ldots, \vec{r}_{2}\right) & =\prod_{i}\left(\sum_{n_{1} n_{2}} C_{n_{1} n_{2}} a_{\xi_{i}}^{\dagger n_{1}} a_{z_{i}}^{\dagger n_{2}}\right) \\
& \times \prod_{i<j}\left(\xi_{i}-\xi_{j}\right)^{m} e^{-\frac{1}{4} \sum_{i}\left(\left|\xi_{i}\right|^{2}+2 z_{i}^{\prime 2}\right)} \\
& \equiv \hat{P}^{\dagger}\left(\vec{r}_{1}, \ldots, \vec{r}_{N}\right)|m\rangle
\end{aligned}
$$

where $|m\rangle$ is the common Laughlin state. To see the incompressibilty, one defines the electron density for this state

$$
\rho_{e}(\vec{r})=\left\langle m\left|\hat{P} \sum_{i} \delta^{3}\left(\vec{r}-\vec{r}_{i}\right) \hat{P}^{\dagger}\right| m\right\rangle /\left\langle m\left|\hat{P} \hat{P}^{\dagger}\right| m\right\rangle .
$$

Since $\left\langle m\left|\left[\hat{P}, \sum_{i} \delta^{3}\left(\vec{r}-\vec{r}_{i}\right)\right]\right| m\right\rangle=D_{r}\langle m \mid m\rangle=0$ (see (8)) , it is easy to show $\rho_{e}(\vec{r})=\left\langle m\left|\sum_{i} \delta^{2}\left(\vec{r}-\vec{r}_{i}\right)\right| m\right\rangle /\langle m \mid m\rangle$, which exactly equals to the density of the Laughlin state for $B_{\|}=0$. Thus, the state is incompressible.

In the same spirit, for the solid state, the variational wave function reads

$$
\Psi_{c}\left(\vec{r}_{i}\right)=\hat{P}^{\dagger} \psi^{c}\left(\xi_{i}\right) e^{-\frac{1}{2} \sum_{i} z_{i}^{\prime 2}},
$$

where $\psi^{c}$ is the variational wave function [10 for $B_{\|}=0$

$$
\begin{aligned}
\psi^{c} & =\exp \left[\frac{1}{4} \sum_{i j} u_{i} B_{i j} u_{j}\right] \\
& \times \exp \left[-\frac{1}{4} \sum_{i}\left(\left|u_{i}\right|^{2}+\left(\bar{\xi}_{i} R_{i}+\xi_{i} \bar{R}_{i}\right)\right]\right],
\end{aligned}
$$

where $u_{i}$ is the site fluctuations of the electrons away from the equilibrium sites $R_{i}$ and $B_{i j}$ are the variational parameters. Similar to Lam and Girvin's, the optimal parameters, in the momentum space, are given by

$$
B_{\vec{q}}=e^{i \theta_{q}} \frac{\omega_{L}(q)-\omega_{T}(q)}{\omega_{L}(q)+\omega_{T}(q)},
$$

where $\theta_{q}$ is a phase factor and $\omega_{L}(q)$ and $\omega_{T}(q)$ are the effective longitudinal and transverse phone frequencies, which are determined by the dynamic matrix $\mathcal{D}$ [10,12]. Since the field is tilted, $\mathcal{D}$ in the present case is related to an effective potential

$$
\begin{aligned}
& V_{\text {eff }}\left(\vec{r}-\vec{r}^{\prime}\right)=\mathcal{P}_{r r^{\prime}} V\left(\vec{r}-\vec{r}^{\prime}\right) \\
& =\left[1-\frac{1}{\mathcal{N}}\left(D_{r}^{\dagger} D_{r}+D_{r^{\prime}}^{\dagger} D_{r^{\prime}}\right)+\frac{1}{\mathcal{N}^{2}} D_{r}^{\dagger} D_{r} D_{r^{\prime}}^{\dagger} D_{r^{\prime}}\right] V\left(\vec{r}-\vec{r}^{\prime}\right), \\
& D_{r}=\sum_{n_{2}=0 ; n_{1}, l=1} C_{n_{1} n_{2}} z_{i}^{\prime n_{2}} C_{n_{1}}^{l}(\bar{\xi} / 2)^{n_{1}-l} \partial_{\xi}^{n_{1}-l},
\end{aligned}
$$

where $\mathcal{N}=\sum_{n_{1} n_{2}} C_{n_{1} n_{2}}^{2}$.

The energies we shall compare for the solid and liquid states are

$$
E_{c, m}=\frac{e^{2}}{\epsilon N}\left\langle\Psi_{c, m}\left|\sum_{i<j} \frac{1}{\left|\vec{r}_{i}-\vec{r}_{j}\right|}\right| \Psi_{c, m}\right\rangle .
$$

Since the analytic single-particle wave function in the symmetric gauge is not ready yet, $C_{n_{1} n_{2}}$ has to be truncated [20]. Fortunately, we find that for the practical value of $\Omega / \omega_{c}$ and a modest tilted angle, the first order approximation, i.e., considering $C_{11}$-contribution only, may be a very good one. The expect energy of the approximation single particle state compares to the exact ground state energy only a few percentage higher [21]. Thus, we consider the subband and the tilted field contribution from $C_{11}$ only.

For the liquid state, we evaluate the energy in (9) by Monte Carlo methods. The detailed simulation technique has been described by Morf and Halperin [9] for the disk geometer. We consider a finite system. The positive charge neutralizing the electron is uniformly distributed on a disk of radius $R_{m}=\sqrt{2 m N} l_{B}$. The energy calculated, at Monte Carlo step $s$, is

$$
E_{N}^{(s)}=\frac{e^{2}}{\epsilon N} \sum_{i, j=1}^{N} \frac{1}{\left|\vec{r}_{i}^{(s)}-\vec{r}_{j}^{(s)}\right|}+\frac{1}{N} \sum_{i=1}^{N} U\left(\vec{r}_{i}^{(s)}\right),
$$

where $\vec{r}_{i}^{(s)}$ is the position of the $i$-th electron in the $s$-th Monte Carlo step; $U\left(\vec{r}_{i}\right)$ is the energy of the $i$-th electron interacting to the neutralizing background: 


$$
U_{B}\left(\vec{r}_{i}\right)=-\frac{e^{2}}{\epsilon} \int_{r \leq R_{m}} \frac{\bar{\rho}_{e}}{\left|\vec{r}-\vec{r}_{i}\right|},
$$

where $\bar{\rho}_{e}=\frac{\nu}{2 \pi l_{B}^{2}}$ is the average electron density. In the Monte Carlo simulation, we have used the unit of the iondisk radius $R_{0}=\left(\pi \bar{\rho}_{e}\right)^{-1 / 2}$. The probability distribution we used in the Monte Carlo simulation has included the tilted field effect, i.e.,

$$
\begin{aligned}
\left|\Psi_{m}\right|^{2} & =\mid \prod_{i}\left(\sum_{n_{1} n_{2}} C_{n_{1} n_{2}}\right) a_{\xi_{i}}^{\dagger n_{1}} a_{z_{i}}^{\dagger n_{2}} \prod_{i<j}\left(\xi_{i}-\xi_{j}\right)^{m} \\
& \times\left. e^{-\frac{1}{4} \sum_{i}\left(\left|\xi_{i}\right|^{2}+2{z_{i}^{\prime}}^{2}\right)}\right|^{2}=e^{-H_{m}}
\end{aligned}
$$

In the real calculation, the perpendicular component $z^{\prime}$ can always integrated out by hands. We identify this energy of the $s$-th Monte Carlo step as the liquid state energy per electron. The simulations were done for the finite systems $N=20,30,42$ and 71 . For $N=20$ and 30 , this energy was computed by 50 independent Monte Carlo simulations, each of which consists of 170,000 Monte Carlo steps. For $N=42$ and 71, the corresponding numbers are 30 and 110,000 . The results for $E_{m}$ are fitted by the polynomials

$$
E_{m} \approx A+B_{1} / \sqrt{N}+B_{2} / N
$$

where and in the following, the energy unit is $e^{2} / \epsilon l_{B}$. We identify the zero-th order coefficient $A$ as the result of thermodynamic limit. The values of $A(\nu)$ of $\theta$ for $\Omega / \omega_{c}=0.8$ and 0.4 are listed in Table I.

Table I(a) The thermodynamic limit of $E_{m}$ for $\Omega / \omega_{c}=0.8$.

\begin{tabular}{ccccc}
\hline \hline$\theta$ & $A(1 / 3)$ & $A(1 / 5)$ & $A(1 / 7)$ & $A(1 / 9)$ \\
\hline 0 & -0.4099 & -0.3275 & -0.2809 & -0.2499 \\
\hline$\pi / 7$ & -0.4131 & -0.3292 & -0.2820 & -0.2509 \\
\hline$\pi / 5$ & -0.4146 & -0.3300 & -0.2825 & -0.2511 \\
\hline$\pi / 4$ & -0.4163 & -0.3307 & -0.2830 & -0.2514 \\
\hline \hline
\end{tabular}

Table I(b) The thermodynamic limit of $E_{m}$ for $\Omega / \omega_{c}=0.4$.

\begin{tabular}{ccccc}
\hline \hline$\theta$ & $A(1 / 3)$ & $A(1 / 5)$ & $A(1 / 7)$ & $A(1 / 9)$ \\
\hline$\pi / 8$ & -0.4151 & -0.3303 & -0.2827 & -0.2512 \\
\hline$\pi / 5$ & -0.4176 & -0.3315 & -0.2835 & -0.2518 \\
\hline$\pi / 4$ & -0.4258 & -0.3356 & -0.2862 & -0.2537 \\
\hline \hline
\end{tabular}

For $\theta=0, A$ is independent of $\Omega / \omega_{c}$, e.g., for $\nu=1 / 3$, we have $A=-0.4099$, which is consistent with results by Levesque et. al [8] and Morf and Halperin [9].

According to the results of the thermodynamic limit, one can figure out the energies dependent on the filling factor $\nu$ and the tilted angle $\theta$. For $\Omega / \omega_{c}=0.8$, one can fit the energy $E_{\theta}(\nu)$ by the following interpolation formulas

$$
\begin{aligned}
& E_{0}=-0.782133 \nu^{1 / 2}+0.166 \nu^{1.241}-0.009 \nu^{2.18} \\
& E_{\frac{\pi}{7}}=-0.782133 \nu^{1 / 2}+0.154 \nu^{1.241}-0.010 \nu^{2.18}
\end{aligned}
$$

$$
\begin{aligned}
& E_{\frac{\pi}{5}}=-0.782133 \nu^{1 / 2}+0.148 \nu^{1.241}-0.009 \nu^{2.18}, \\
& E_{\frac{\pi}{4}}=-0.782133 \nu^{1 / 2}+0.144 \nu^{1.241}-0.017 \nu^{2.18} .
\end{aligned}
$$

For $\Omega / \omega_{c}=0.4$, the fitting results are

$$
\begin{aligned}
& E_{\frac{\pi}{8}}=-0.782133 \nu^{1 / 2}+0.146 \nu^{1.241}-0.010 \nu^{2.18}, \\
& E_{\frac{\pi}{6}}=-0.782133 \nu^{1 / 2}+0.138 \nu^{1.241}-0.015 \nu^{2.18}, \\
& E_{\frac{\pi}{4}}=-0.782133 \nu^{1 / 2}+0.110 \nu^{1.241}-0.026 \nu^{2.18} .
\end{aligned}
$$

The first one in (14) is corresponding to $\theta=0$, which is consistent with the result of Levesque et al [8]. One observes that the exponents in the fit formulas are not dependent on the tilted field; Neither are almost the coefficients of $\nu^{2.18}$ except in the last one of (15) which may be because the approximation single-particle wave function is a little bit poor in this parameter $(\Delta \varepsilon / \varepsilon \sim 13 \%)$.

Now, we turn to the electron solid. The strategy we take is the same as Lam and Girvin's [10]: Expanding the Coulomb interaction to the second order of the fluctuation $u_{i}$ and determining the variational parameter $B_{i j}$. Then, calculate the energy (9) exactly. To the harmonic approximation, the harmonic term $E_{c, h}$ of the energy is given by

$$
\begin{aligned}
E_{c, h} & =\frac{m}{N} \sum_{q}\left(\omega_{T}(q)+\omega_{L}(q)\right)^{2} \\
& =\frac{m}{N} \sum_{q}(\operatorname{Tr} \mathcal{D}(q)+\sqrt{4 \operatorname{Det} \mathcal{D}(q)}),
\end{aligned}
$$

where $\mathcal{D}$ is the effective dynamic matrix and $\vec{q}$ is the wave vector in the Brillouin zone. Comparing to the uncorrelated energy $E_{u c, h}=\frac{2 m}{N} \sum_{q}\left(\omega_{T}^{2}(q)+\omega_{L}^{2}(q)\right)$, the fluctuation is lowered the energy because the transverse modes are favored while the longitudinal modes are disfavored [10.

The numerical results of $E_{\theta}(\nu)$ are as follows. For $\Omega / \omega_{c}=0.8$, one has

$$
\begin{aligned}
& E_{0}=-0.782133 \nu^{1 / 2}+0.2430 \nu^{3 / 2}+0.1610 \nu^{5 / 2}, \\
& E_{\frac{\pi}{7}}=-0.782133 \nu^{1 / 2}+0.2200 \nu^{3 / 2}+0.1598 \nu^{5 / 2}, \\
& E_{\frac{\pi}{5}}=-0.782133 \nu^{1 / 2}+0.1971 \nu^{3 / 2}+0.1596 \nu^{5 / 2}, \\
& E_{\frac{\pi}{4}}=-0.782133 \nu^{1 / 2}+0.1728 \nu^{3 / 2}+0.1594 \nu^{5 / 2} .
\end{aligned}
$$

For $\Omega / \omega_{c}=0.4$, it is

$$
\begin{aligned}
& E_{\frac{\pi}{8}}=-0.782133 \nu^{1 / 2}+0.1926 \nu^{3 / 2}+0.1595 \nu^{5 / 2}, \\
& E_{\frac{\pi}{6}}=-0.782133 \nu^{1 / 2}+0.1619 \nu^{3 / 2}+0.1593 \nu^{5 / 2}, \\
& E_{\frac{\pi}{4}}=-0.782133 \nu^{1 / 2}+0.0992 \nu^{3 / 2}+0.1592 \nu^{5 / 2},
\end{aligned}
$$

The first one in (18) corresponds to the energy of $\theta=0$, which is consistent with Lam and Halperin's result [10]. We see that again the coefficients of $\nu^{5 / 2}$ are almost not affected by the tilted field.

Using the fitting results (14) and (18), (15) and (19), one can obtain the estimated critical filling factors of $\theta$ (Table II). 
Table II(a) The critical filling factors for $\Omega / \omega_{c}=0.8$.

\begin{tabular}{ccc}
\hline \hline$\theta$ & $\Delta E_{\theta}(1 / 3)$ & $\nu_{c}$ \\
\hline 0 & -0.015 & 6.63 \\
\hline$\pi / 7$ & -0.013 & 6.45 \\
\hline$\pi / 5$ & -0.010 & 5.58 \\
\hline$\pi / 4$ & -0.007 & 4.54 \\
\hline \hline
\end{tabular}

Table II(b) The critical filling factors for $\Omega / \omega_{c}=0.4$.

\begin{tabular}{ccc}
\hline \hline$\theta$ & $\Delta E_{\theta}(1 / 3)$ & $\nu_{c}$ \\
\hline$\pi / 8$ & -0.009 & 5.46 \\
\hline$\pi / 6$ & -0.007 & 4.45 \\
\hline$\pi / 4$ & -0.002 & 3.46 \\
\hline \hline
\end{tabular}

In Table II, $\Delta E_{\theta}(1 / 3)$ is the energy difference between the liquid and solid at $\nu=1 / 3$, whose magnitude reduces considerably as the tilted angle increases from 0 to $\pi / 4$. In this work, we did not check the Laughlin-Jastrow correlation to the electron solid. It has been pointed out that the solid may be unstable at the exact $\nu=1 / 5$ due to this correlation [15]. Thus, our variational calculation may not be enough to completely understand the physics at the exact odd denominator filling factor $1 / \mathrm{m}$. Due to the instability, the Hall plateau may not disappear. However, our calculation indicates that the insulating phase may harder be melt as $\nu \rightarrow 1 / 5$ such that the width of the valley of the longitudinal resistance may become narrow as the tilted angle increases.

On the other hand, although our result did not show the critical filling factor is shifted to near $1 / 3$, the magnitude of $\Delta E_{\theta}(1 / 3)$ reduces remarkably as the field is tilted. According to Ref. [12], after accounting the Laudau level mixing, the energies of the solid and liquid states for $\theta=0$ are lowered about $0.8 \%$ and $0.1 \%$ respectively at $\nu \sim 1 / 3$ and $r_{s}=\frac{e^{2} / \epsilon R_{0}^{2}}{\nu \hbar \omega_{c} / 2} \sim 2$. The lowering of the energy of the solid is larger than that of the liquid about $0.002 \frac{e^{2}}{\epsilon l_{B}}$, which can nearly overcome the energy difference $\Delta E_{\theta}(1 / 3)$ for large angles $\theta$ in Table II. If one assumes the energy lowering due to the Laudau level mixing is independent of the tilted field and considers the energy of the liquid state for $\nu$ being nearly but not exactly $1 / 3$ is in fact higher than our interpolating estimation [16], it is possible that at a large tilted angle, the energy of the solid becomes lower than that of liquid for $\nu \sim 1 / 3$. Thus it may be anticipated that in the vicinity of $\nu=1 / 3$ for the electron system in the presence of the tilted field, the insulating phase may be observed for a larger tilted angle. To verify this expected phenomenon, both further experimental and numerical works are required.

The authors were grateful to the discussions with $\mathrm{R}$. R. Du, Z. B. Su and F. C. Zhang. This work is partially supported by NSF of China.
[1] D. C. Tsui, H. L. Stormer, and A. C. Gossard, Phys. Rev. Lett. 48, 1559 (1982).

[2] For a recent review, see T. Chakraborty, Adv. Phys. 49, 959 (2000).

[3] I. B. Spielman, J. P. Eisenstein, L. N. Pfeiffer and K. W. West, Phys. Rev. Lett. 84,5808 (2000); ibid 87, 036803 (2001).

[4] W. Pan, R. R. Du, H. L. Stormer, D. C. Tsui, L. N. Pfeiffer, K. W. Baldwin and K. W. West, Phys. Rev. Lett. 83, 820 (1999); M. P. Lilly, K. B. Cooper, J. P. Eisenstein, L. N. Pfeiffer and K. W. West, Phys. Rev. Lett. 83, 824 (1999).

[5] D. A. Syphers and J. E. Furneaux, Solid Stat. Commun. 65, 1513 (1988).

[6] V. Halonen, P. Pietilainen and T. Chakraborty, Phys. Rev. B 41, 10202 (1990).

[7] R. B. Laughlin, Surf. Sci. 142, 153 (1984); T. Chakraborty, Phys. Rev. B 31, 4026 (1985).

[8] D. Levesque, J. J. Weis and A. H. MacDonald, Phys. Rev. B 30, 1056(1984).

[9] R. Morf and B. I. Halperin, Phys. Rev. B 33, 2221 (1986).

[10] P. K. Lam and S. M. Girvin, Phys. Rev. B 30, 473 (1984).

[11] M. B. Santos, Y. W. Suen, M. Shayegen, Y. P. Li, L. W. Engel and D. C. Tusi, Phs. Rev. Lett. 68, 1188 (1992); M. B. Santos, J. Jo, Y. W. Suen, L. W. Engel and M. Shayegen, Phys. Rev . B 46, 13639 (1992).

[12] X. Zhu and S. G. Louie, Phys. Rev. Lett. 70, 335 (1993); R. Price, P. M. Platzman and S. He, Phys. Rev. Lett. 70, 339 (1993).

[13] M. Shayegan, J. Jo, Y. W. Suen, M. Santos, V. J. Goldman, Phys. Rev. Lett. 65, 2916 (1990); S. He, F. C. Zhang, X. C. Xie, and S. Das Sarma, Phys. Rev. B 42, 11376 (1990).

[14] R. Price, X. Zhu, S. Das Sarma and P. M. Platzman, Phys. Rev. B 51, 2017 (1995).

[15] L. Zheng and H. A. Fertig, Phys. Rev. Lett. 73, 878 (1994).

[16] B. I. Halperin, Phys. Rev. Lett. 52, 1583 (1984); 52, 2390 (E) (1984).

[17] T. Sajoto, Y. P. Li, L. W. Engel, D. C. Tsui and M. Shayegan, Phys. Rev. Lett. 70, 2321 (1993).

[18] J. C. Maan, Two-Dimensional Systems, Heterostructures and Superlattices, edited by G. Bauer. F. Kurcher and H. Heinrich (Heidelberg: Springer,1984).

[19] Y. Yu, S.J. Yang and Z. B. Su, Phys. Rev. B 62, 15371(2000). Shi-Jie Yang, Yue Yu and Jin-Bin Li, Phys. Rev. B 65, 073302 (2002).

[20] Note that a truncated state is still incompressible with the same density as that of the corresponding Langhlin state.

[21] For examples, for $\Omega / \omega_{c}=0.8$, one has $\Delta \varepsilon / \varepsilon \sim 0.3 \%$ for $\theta=\pi / 8$ and $3.6 \%$ for $\theta=\pi / 4$; For $\Omega / \omega_{c}=0.4$, it is $3.1 \%$ for $\theta=\pi / 7$ and $4.9 \%$ for $\theta=\pi / 6$. As a comparison, if one uses the zero tilted field wave function, i.e., $C_{11}=0$, one has $\Delta \varepsilon_{0} / \varepsilon \sim 3.0 \%$ for $\theta=\pi / 8$ and $11.0 \%$ for $\theta=\pi / 4$ for $\Omega / \omega_{c}=0.8$; For $\Omega / \omega_{c}=0.4$, it is $9.8 \%$ for $\theta=\pi / 7$ and $12.4 \%$ for $\theta=\pi / 6$. 